\title{
Viscosity Control for Extrusion Processes Using Recycled Polymers
}

\author{
Bao Kha Nguyen, Gerard McNally, Alan Clarke
}

\begin{abstract}
The aim of this paper is to develop a new extruder control system for recycled materials which has ability to automatically maintain constant a polymer melt viscosity of mixed recycled polymers during extrusion, regardless of variations in the Melt Flow Index (MFI) of recycled mixed grade high density polyethylene (HDPE) feedstock. A closed-loop controller is developed to automatically regulate screw speed and barrel temperature profile to achieve constant viscosity and enable consistent processing of variable grade recycled HDPE materials. The experimental results of real time viscosity measurement and control using a 38mm single screw extruder with different recycled HDPEs with widely different MFIs are reported in this work.
\end{abstract}

Keywords-viscosity, control, extrusion, recycled polymers

\section{Introduction}

Plastics recycling and reducing the volume of plastic waste going to landfill is an emotive subject, highly publicised by our increasing dependence on oil, fears over future supplydemand and concern for our environment. Social responsibility has encouraged the plastics industry to invest in technologies to minimise the disposal of plastic waste to landfill through recycling processes.

Recycling process of plastic bottles normally comprises of sorting, washing and pelletizing processes. Despite considerable development in waste plastic sorting technology (1-2), the properties and quality of the recycled polymers are normally lower than those of the virgin material (3) and strongly dependent on the operating conditions (4).

During the extrusion process, the recycled polymers undergo a complex thermomechanical transformation, inducing complex rheological behaviour of recycled polymer melts. The processing conditions can have a major impact on the polymer as its molecular structure may undergo change as a result of chemical reactions (oxidation, cross-linking) taking place in the extruder, which are usually induced by high temperature and high shear stress conditions. The change in molecular structure brought about by degradation can significantly affect the melt flow properties and alter the physical and chemical properties of the recycled materials and the aesthetic properties of polymer products.

Bao Kha Nguyen, Alan Clarke

Queen's University Belfast

United Kingdom

Gerard McNally

Northern Ireland Polymers Association

United Kingdom
In comparison with melt temperature and pressure, melt viscosity is largely recognized as the most relevant indicator of melt quality because it is directly related to the aesthetic/dimensional properties of the melt and the molecular orientation relating to the functional properties of a polymeric extrudate (5). With recycled plastic, the main issues for extrusion processes are the variations in viscosity or in MFI of the recycled polymer feedstock (6). These variations of recycled plastic between batches of recycled materials are greater than for virgin materials due to differences in the physical properties of the source of waste plastic materials used. As a result, it is difficult to process these into good quality products without incurring high scrap levels, for instance, if the viscosity is too high, the extruded products will collapse on exit from the extruder die, however if it is too low, the material will not flow properly through the die resulting clogging at the die. Both result in extrusion processes downtime, material wastage through the generation of scrap and the variable performance with regard to product quality. Real time monitoring and control of the melt viscosity in the polymer flow are therefore desirable to achieve high product quality in the extrusion processes.

Several types of melt viscosity measurement techniques have been developed recently including off-line laboratory capillary rheometry, torsional viscometry (7), on-line sidestream (8)...However there have been few reports on the application of real time viscosity control in extrusion processes and this is probably due to design complexity, the development of meaningful control strategies, measurement accuracy and high cost. An alternative approach which has also been reported, is to determine the melt viscosity, based on modelling techniques. The development of combining mechanistic and parametric data in grey box modelling techniques, associated with intelligent algorithms such as neural network and genetic algorithms, have also been reported (9-11). However, this research is only at a modelling stage, and there has been no reports on the application of these models to real time control systems.

This paper describes the introduction of a total integration system, including viscosity measurement and control for recycled polymers during the extrusion processes, using an automatic closed - loop control technology. This innovation will enable constant melt viscosity to be maintained during the extrusion of mixed grade polymers, regardless of variations in MFI of feed materials and enable consistent processing of variable grade recycled plastic. All the experiments described in this paper were carried out on a $38 \mathrm{~mm}$ single screw extruder with slit die. The extrusion system was also fitted with a gravimetric loss-in-weight hopper feeder for real time throughput measurement. Using this device, the melt viscosity 
can be calculated online simply from the measurement of throughput $(\mathrm{kgs} / \mathrm{hr})$ and the pressure drop through the die using the geometric dimensions of the extruder/die arrangement (12). Both the screw speed and the first three barrel temperatures zones are used as control variables and automatically regulated with respect to variations in the melt viscosity of feed materials during the extrusion process, by the novel control system.

The paper is organized as follows. Section 2 presents the experimental setup for viscosity control system. This includes the use of the loss-in-weight hopper feeder system to monitor the throughput of material consumption during the process. The calculation of viscosity in real time based on throughput and pressure drop through the die compared with offline viscosity measurement using laboratory rheometry technique is shown in section 3. The variation in viscosity of recycled polymers at a fixed extruder screw speed using open loop control is reported and discussed in this section. In section 4, the closed-loop control structure for viscosity control of mixed MFI polymers is described along with experimental results obtained from real time viscosity control of different grades of recycled HDPE polymers. Concluding remarks are provided in Section 5.

\section{Experimental setup}

The extrusion trials were carried out using a $38 \mathrm{~mm}$ Killion $\mathrm{KN}-100$, single screw extruder. The barrel comprised three heating zones (feed zone, melting zone, and metering zone) which were controlled by Eurotherm 808 PID controllers. The feed section is also water- cooled to prevent premature rise in polymer temperature. The photograph in Fig. 1 shows the experimental setup comprising an in-line rheometer die for viscosity measurement. This fitted to end of the barrel just prior die, by means of adaptor and clamp rings. The throughput was continually measured by a loss - in - weight hopper feeder equipment manufactured by TSM Control Systems (Fig. 2). This system comprises two hoppers the upper one, for material feeding, and a load cell is used to measure the weight of the material in the lower hopper. The throughput is calculated from the weight loss per unit time of material in the lower hopper. Conventional sensing elements

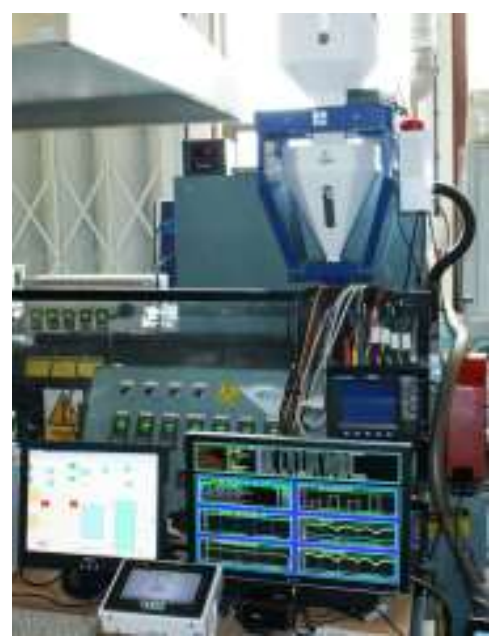

Fig. 1 Experimental apparatus

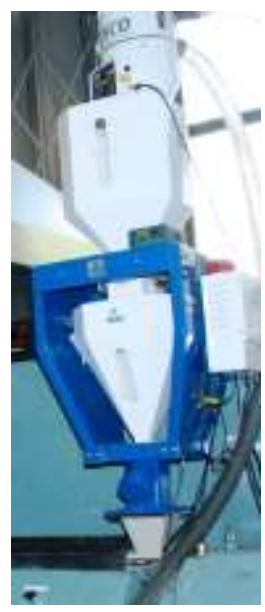

Fig. 2 Loss - in - weight hopper

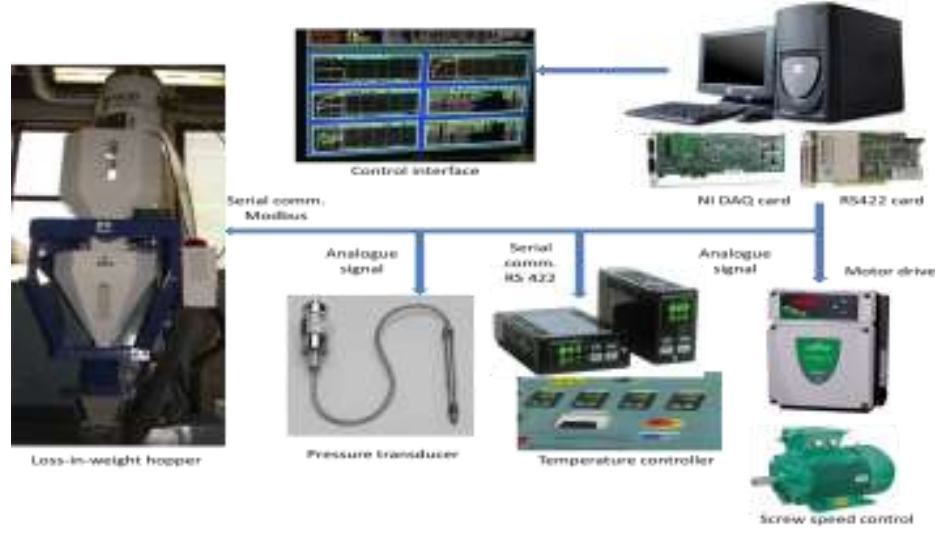

Fig. 3 Data acquisition and communication methods

such as thermocouples and pressure transducers (Dynisco, full scale rating of $34.47 \mathrm{MPa}$ and $20.68 \mathrm{MPa}$ ) are located in the barrel and the die to provide continuous data on the polymer characteristics during extrusion. A schematic diagram of the data acquisition and control system and communication methods used in the experimental apparatus are described in Fig. 3.

Analogue signals from pressure transducers and screw speed are fed to the computer via A/D channels of a PCI6031E Card (National Instruments Inc.). The screw speed was also controlled by a D/A channel and the Quantium III DC motor drive (Control Technique). The barrel zone temperatures were monitored and controlled from the computer through a RS422 communication, using a specific Eurotherm 808 protocol. Throughput from the TSM Loss - In Weight hopper was also monitored and controlled by the computer using Modbus RTU protocol. Serial communications between the devices and the computer were implemented using a Quatec QSC 200/300 PCI card. The sampling time was set to $0.05 \mathrm{~s}$ in all experiments. The system was controlled in real - time using Matlab xPC Target Toolbox.

\section{Viscosity measurement}

In this work, a slit die in-line rheometer was designed and fitted to the exit of the extruder for in-line monitoring of the melt viscosity at the die (Fig.4). This particular method is very relevant to extrusion process control since the instrument is mounted directly in the main melt stream of the extrusion process.. Pressure transducers are located along the length of the capillary and die for pressure drop measurement

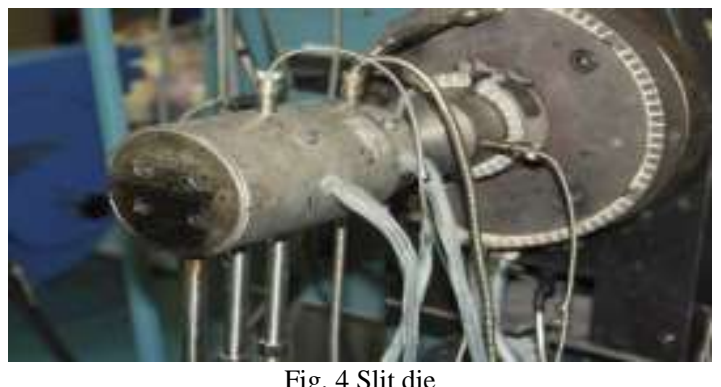

Fig. 4 Slit die 
Proc. of the Second Intl. Conf. on Advances in Mechanical and Automation Engineering - MAE 2015

Copyright $(\odot$ Institute of Research Engineers and Doctors, USA .All rights reserved.

ISBN: 978-1-63248-045-3 doi: 10.15224/ 978-1-63248-045-3-46

(a) Screw speed

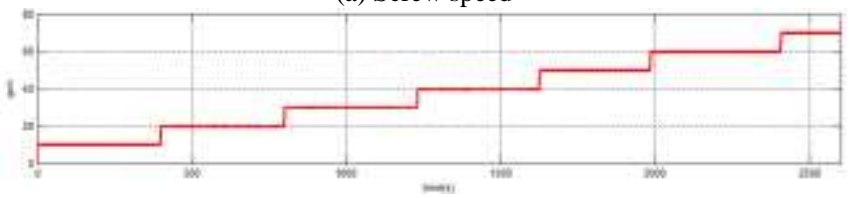

(b) Pressure drop

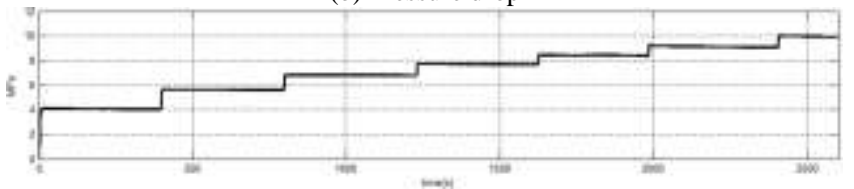

(c) Hopper weight

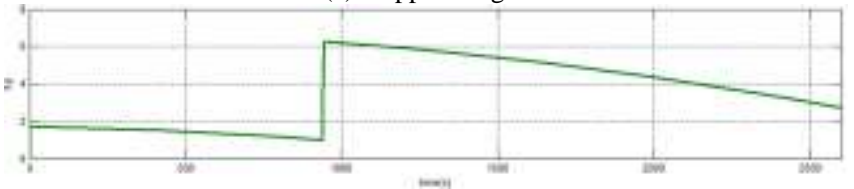

(d) Throughput

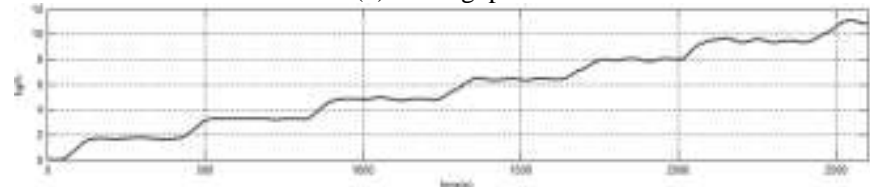

(e) Viscosity

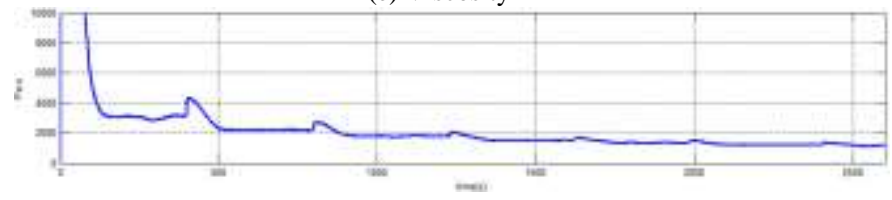

Fig. 5 Experiment for in - line viscosity measurement of virgin HDPE Marlex TR 144

The melt viscosity was determined as being the ratio of the shear stress (calculated from the pressure drop along the inline die) to the shear rate (determined from the throughput) [12] and calculated as follows:

$$
\eta=\frac{W H^{3} \Delta P}{8 L Q}\left[\frac{3 n}{2 n+1}\right]
$$

where $\mathrm{H}$ - Height of the slit, $\mathrm{L}-$ The distance between the pressure transducers, $\Delta \mathrm{P}$ : pressure drop through the die, $\mathrm{Q}$ volumetric flow rate, $\mathrm{W}$ - the width of the slit, $\mathrm{n}$ is the power law index determined from Rheological data of the material

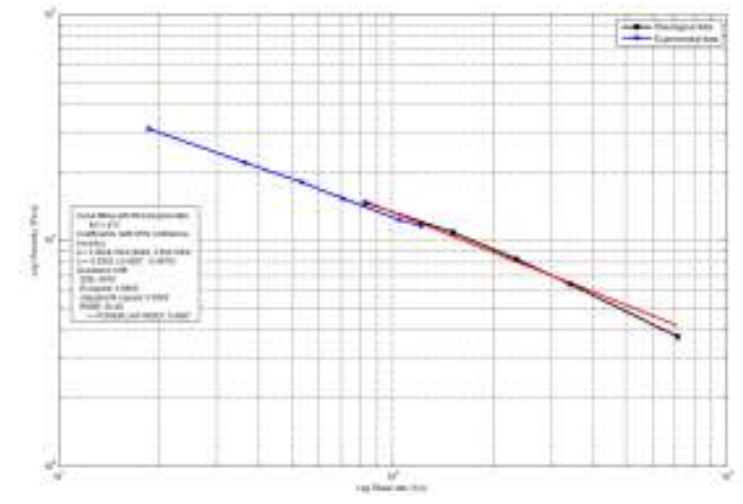

Fig. 6 Experiment and laboratory rheology of virgin HDPE Marlex TR 144 at $200^{\circ} \mathrm{C}$

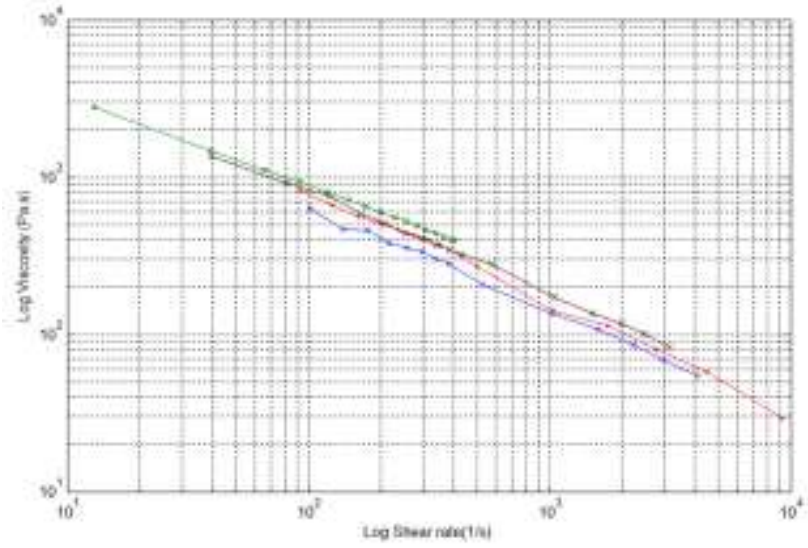

Fig. 7 Laboratory rheology of recycled polymers with different MFI used.

In order to demonstrate the effectiveness of this method of viscosity measurement, experimental viscosity data was obtained using a virgin high density polyethylene (HDPE) Marlex HHM20 TR144, prior to applying it to recycled materials. The results were compared with viscosity data measured using a Rosand dual-capillary rheometer. The same temperature was used for both tests. A curve fitting procedure was applied to determine the power law index value $n=0.4097$ from the rheological data of this material.

During the experiment, the effect of step - changes in screw speed (Fig. 5a) on the dynamic response of the in-line measurement was investigated. The pressure drop across the die (P1-P3) is shown in Fig. 5b. Actual extruder throughput weight of material in the hopper is recorded and when the weight of material in the hopper is less than $1 \mathrm{~kg}$, an automatic pneumatic valve is activated to allow more material to be fed into the hopper (Fig. 5c) and closed if it is more than $6 \mathrm{~kg}$. Extruder throughput is calculated from the loss in weight in this hopper and is shown in Fig. 5d. With an assumption that the amount of material entering and leaving the extruder is the same, the viscosity calculated from this thoughput and pressure drop data is shown in Fig. 5e. The viscosity is calculated by this method is compared with the rheological data from the laboratory capillary rheometer and was found to give comparable results (Fig. 6). Although there is a small offset error as the in-line measurement is a bit lower than viscosity determined offline using laboratory capillary rheometer, both readings are in good agreement in slope.

Offline viscosity measurement using laboratory rheometer for different batches of recycled HDPE polymers supplied by Cherry Polymers, Northern Ireland were also carried out to obtain the average power law index of these materials, $\bar{n}=0.3868$, and shown in Fig.7. Five recycled polymers with different MFIs (at $190^{\circ} \mathrm{C} / 2.16 \mathrm{~kg}$ ) collected in different batches at different times at the company was used in this experiment, some of the materials are shown in Fig. 8. The recycled materials are produced mainly from post-consumer plastic bottles and grade range from 0.1914 to 0.6466 MFI. Both materials with the lowest and highest MFI in this range were used in the test. 

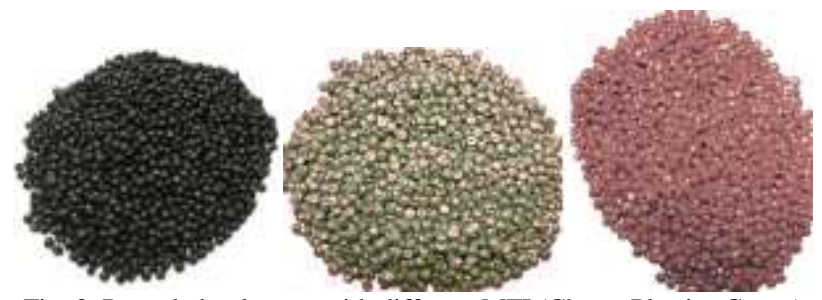

Fig. 8. Recycled polymers with different MFI (Cherry Plastics Group)

Replacing die dimension and the average power law index value to (1), the viscosity for recycled materials is then calculated using the following equation:

$$
\eta=\frac{W H^{3} \Delta P}{8 L Q}\left[\frac{3 n}{2 n+1}\right]
$$

where $\mathrm{q}$ is the throughput $(\mathrm{kg} / \mathrm{h})$

The result of online viscosity calculation using the above equation is shown in Fig. 9. The screw speed (Fig. 9a) was

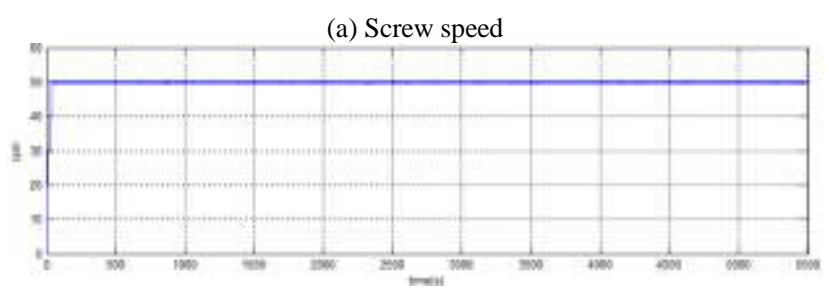

(b) Pressure

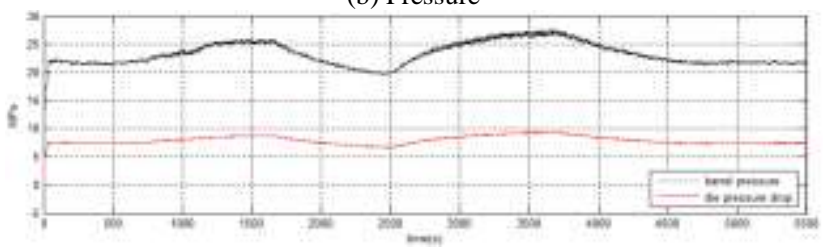

(c) Throughput

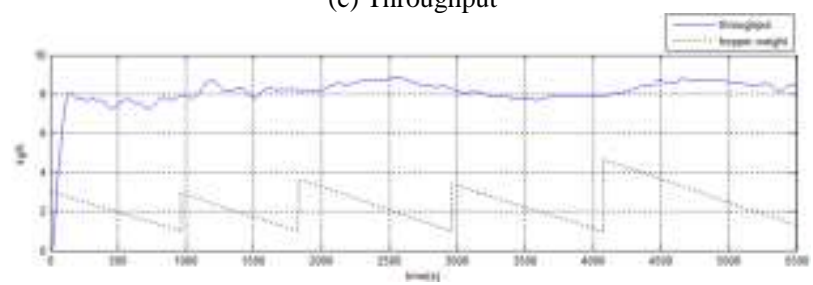

(d) Viscosity

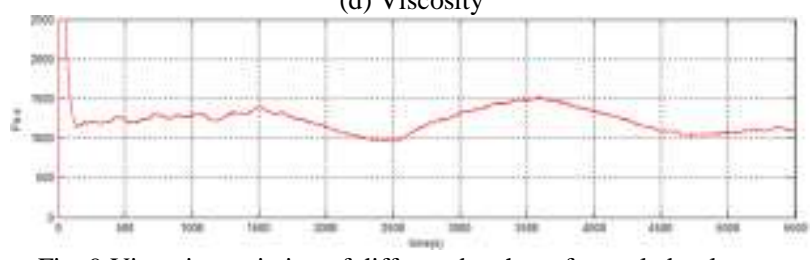

Fig. 9 Viscosity variation of different batches of recycled polymers

kept constant at 50rpm and different feed materials were charged into the hopper at random points throughout the experiment. The barrel pressure and pressure drop through slit die (P1-P3) are shown in Fig. 9b. The throughput and actual weight of material in the hopper are shown in Fig. 9c. The

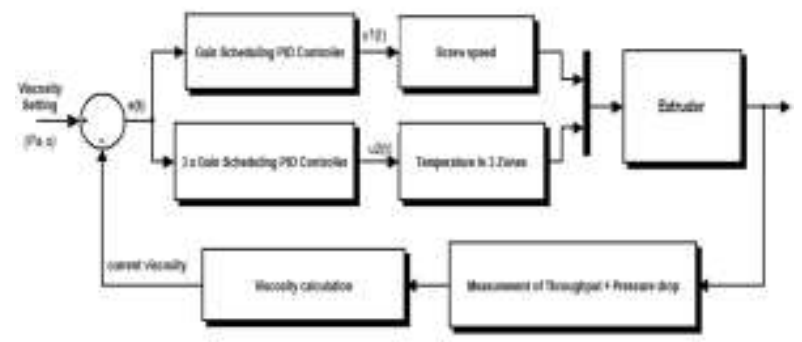

Fig. 10 Diagram of closed loop viscositv control

variations of material weight in the hopper show the change of different feed materials. The melt viscosity of the various recycled polymers in the die was calculated using equation (6) and is shown in Fig. 9d. As the results show, the variation in viscosity of different batches of recycled materials due to the inconsistency in the physical properties of the recycled feedstock in this experiment is significantly high, approximate 500pa.s (max). This will result in difficulties processing of these polymers and will currently require manual intervention by skilled operators to change the machine processing parameters in most of industrial extruders.

In order to improve the product quality and minimise the reliance on manual operation in extrusion processes, a closedloop controller is developed to automatically regulate screw speed and temperature to achieve constant viscosity during extrusion process and enable consistent processing regardless of polymer grade of the recycled plastic materials.

\section{Closed - loop viscosity control}

In this present work however, both the screw speed and the barrel temperature are used as control variables in the controller design. As the combination of gain scheduling technique and conventional PID controller has been used widely in industrial applications and has proven to be stable in many industrial plants, this control strategy was applied to maintain constant melt viscosity regardless the change in MFI of feed materials in this research. The block diagram of the proposed controller is shown in Fig. 10. Input of both controllers in this diagram is the error between the desired and actual viscosity values calculated from the measurement of throughput and pressure drop through the die using (Eq. 6). The screw speed and temperature obtained by the conventional PID algorithm are the outputs of controller 1 and 2, respectively:

$$
u_{1,2}(t)=K_{p} e(t)+K_{i} e(t) d t+K_{d} \frac{d e(t)}{d t}
$$

where $K_{p}, K_{i}, K_{d}$ are PID gains. These parameters were changed using gain scheduling technique. To allow better heat effect to polymer melt, temperature in Zone 1, 2, and 3 are changed with the same strategy.

\section{Experimental results}

Figure 11 shows the control performance of the viscosity control for extrusion process using recycled polymers. Different materials in low and high MFI shown in section 3 
were fed into the hopper at random points throughout this experiment. Viscosity is set at 1500pa.s. Set and current

(a) Viscosity

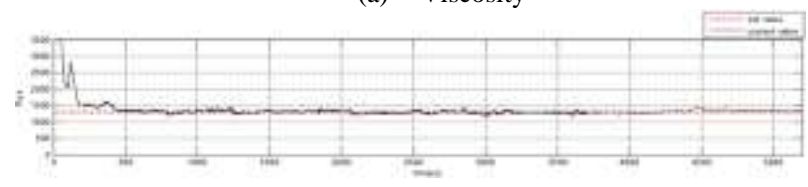

(b) Pressure

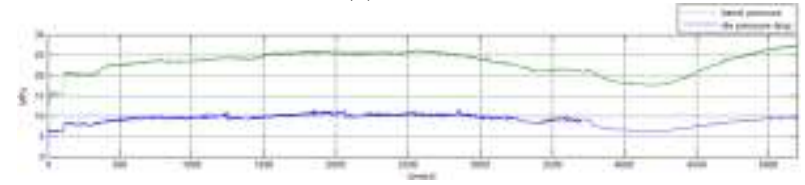

(c) Throughput

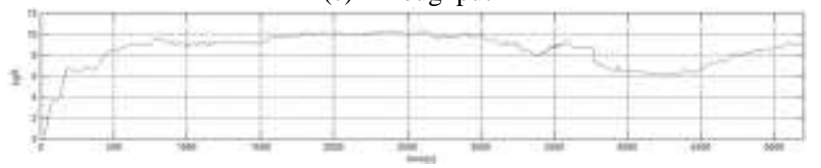

(d) Temperature

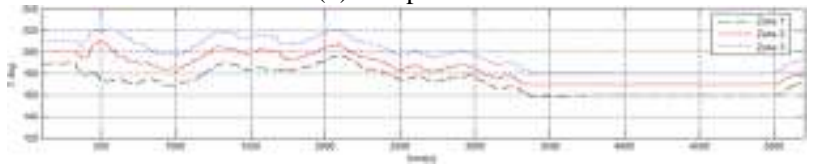

(e) Screw speed

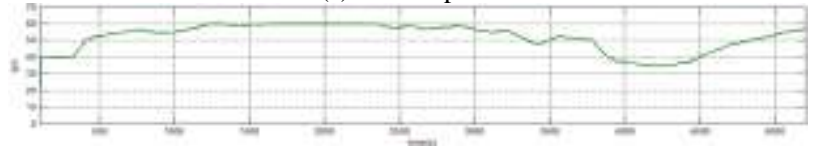

(f) Motor current

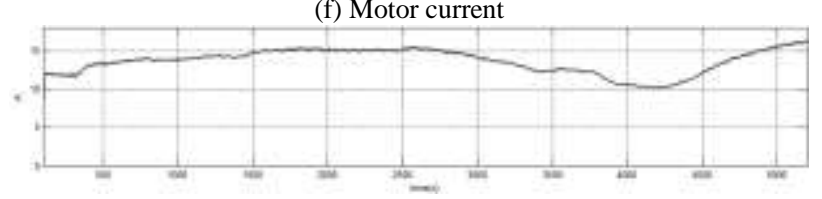

Fig. 11 Closed loop viscosity control for recycled polymers

viscosity values are shown in Fig. 11a. Due to chemical reactions taking place in the extruder under high temperatures and mechanical stresses, the change in molecular structure of recycled polymers may cause the degradation and reduce the quality of extruded products. To avoid this degradation, the controller only regulated the temperature (Fig. 11d) and screw speed (Fig. 11e) in specific ranges. Temperature ranges were set to $\left[160^{\circ} \mathrm{C}-200^{\circ} \mathrm{C}\right],\left[170^{\circ} \mathrm{C}-210^{\circ} \mathrm{C}\right]$ and $\left[180^{\circ} \mathrm{C}-220^{\circ} \mathrm{C}\right]$ for Zone1, Zone 2 and Zone 3, respectively. Barrel pressure and pressure drop through the die in Fig. 11b reduced significantly when high MFI material (low in viscosity) was fed into the hopper at about $300^{\circ} \mathrm{s}$. However, the controller automatically adjusted the temperature in Zone 1, 2, 3 and screw speed to bring the viscosity close to the set value. Throughput in Fig. $11 \mathrm{c}$ and motor current (Fig. 11f) are also decreased with this low viscosity recycled material. It can be observed from this figure that the current viscosity is closed with the desired value irrespective of the change of different feed materials. Therefore, the proposed control algorithm proves to be effective for viscosity control of the recycled polymers in the extrusion processes.

\section{Conclusions}

In this paper, the variations in melt viscosity of mixed grade recycled polymers during the extrusion process have been investigated. The work reports on real time viscosity measurement calculated from actual throughput and pressure drop through the die during extrusion. A conventional closed loop PID controller incorporated with a gain scheduling technique was applied to control the viscosity of polymer melt during extrusion by automatically altering the screw speed and the temperature in the first three zones of the barrel. Experimental evaluation shows that the proposed controller adaptively maintains the viscosity of the melt flow in the die regardless of the differences in viscosity of the feed materials used. This development has significant relevance to extrusion process applications and enables the melt flow properties of different grades of polymer to be processed consistently similar to extrusion of virgin polymer, thus allowing mixed grade polymer feedstock to be processed in a more consistent manner.

\section{Acknowledgment}

This work was supported by the European Seventh Framework Programme

\section{References}

[1] Tachwali Y, Al-Assaf Y, Al-Ali AR. Automatic multistage classification system for plastic bottlesrecycling. Resour Conserv Recy 2007, 52 (2), pp. 266-285.

[2] Kenny GR, Bruner RS. Experience and advances in automated separation of plastics for recycling. J Vinyl AdditTechn 1994, 16 (3), pp. 181-186.

[3] Javierrea C, Claveríaa I, Ponza L, Aísaa L, Fernándeza A. Influence of the recycled material percentage on the rheological behaviour of HDPE for injection moulding process. Waste Manage 2007, 27 (5), pp. 656663.

[4] Loultcheva MK, Proietto M, Jilov N, La Mantia FP. Recycling of high density polyethylene containers. Polym Degrad Stab 1997, 57 (1), pp. 77-81.

[5] McAfee M, Thompson S. A novel approach to dynamic modelling of polymer extrusion for improved process control. Proceedings of the Institution of Mechanical Engineers, Part I, J Systems Control 2007, 221 (4), pp. 617-628.

[6] Shenoy AV, Saini DR, Nadkarni VM. Estimation of the melt rheology of polymer waste from melt flow index. Polymer 1983; 24 (6): 722-728.

[7] Forgacs RL. Improved Torsion Viscometer for Polymer Studies. Rev Sci Instrum 1965, 36 (3), pp. 307-313.

[8] Dealy JM, Broadhead TO. Process rheometers for molten plastics: a survey of existing technology. Polym Eng Sci 1993, 33(23), pp. 15131523.

[9] Liu XQ; Li K; McAfee M, Deng J. 'Soft-sensor' for real-time monitoring of melt viscosity in polymer extrusion process. The 49th IEEE Conf. Decision and Control 2010, pp. 3469 - 3474.

[10] McAfee M, McNally G. Real-time measurement of melt viscosity in single-screw extrusion. T I Meas Control 2006, 28 (5), pp. 481-497.

[11] McAfee M, Thompson S. A soft sensor for viscosity control of polymer extrusion. European Control Conference, Greece, 2007.

[12] Son YG. Determination of shear viscosity and shear rate from pressure drop and flow rate relationship in a rectangular channel. Polymer 2007 48, pp. 632-637. 